\title{
Thermal regimes in solar-thermal linear collectors
}

\author{
Javier Muñoz ${ }^{*}$ José M. Martínez-Val, Rubén Abbas \\ Grupo de Investigaciones Termoenergéticas ETSII- Madrid Polytechnic University \\ * Corresponding author. Tel: +34 913363128, Fax: +34 913363079,E-mail: jamunoz@etsii.upm.es
}

\begin{abstract}
In this paper the physics of linear receivers is analyzed. This analysis is oriented to evaluate the dependence of the thermal performance of the receiver on the characteristics of the concentrated solar radiation. It will be seen that two thermal regimes can be distinguished in that dependence, what will point out the need of studying at depth the features of systems with moderate concentration factors.

A fundamental question can be formulated as follows: what is the concentration factor required for obtaining the best solar energy exploitation within a design window established by some technical constraints?

In this performance analysis it has been found that moderate concentration factors (about 20 to achieve $400^{\circ} \mathrm{C}$ ) can be close to optimal in a cost benefit analysis, taking into account temperature constraints. Beyond these values, thermal and exergetic efficiency curves advice to limit the concentration factors to values that could be achievable by linear Fresnel reflecting collectors, avoiding mobile parts such as ball-joints or flexible joints that could be important leak-points, as well as metal-glass welds, which are another cause of failure in trough collectors
\end{abstract}

Keywords: Renewable energy, Concentrated solar energy, Linear receiver, Fresnel

\section{Nomenclature}

$\alpha \quad$ selective coating absorptivity.....

$C_{f}$ concentration factor

DNI direct normal radiation

$\varepsilon \quad$ selective coating emissivity.

$\eta \quad$ efficiency.....

$\eta_{e x}$ exergetic efficiency.

$\eta_{\text {th }}$ thermal efficiency

$\eta_{\text {th } \rightarrow e}$ thermo-electrical conversion efficiency.-

$h \quad$ heat transfer coefficient ................ W/ $\mathrm{m}^{2} \cdot \mathrm{K}$

$Q_{\text {conv }}$ convection thermal losses. $\mathrm{W} / \mathrm{m}^{2}$
$Q_{\text {rad }}$ radiation thermal losses .................... W/ $\mathrm{m}^{2}$

$Q_{\text {sun }}$ available thermal power.................... W/ $\mathrm{m}^{2}$

$Q_{\text {use }}$ transferred power to the fluid............. W/ $\mathrm{m}^{2}$

$T_{\mathrm{a}} \quad$ bulk temperature of the air .......................

$T_{\mathrm{c}}$ absorbing surface temperature .................

$T_{\text {env }}$ environment temperature .........................

$T_{f} \quad$ heat carrier temperature ...........................

$T_{\text {grnd }}$ ground temperature ................................

$T_{\text {sky }}$ high atmosphere temperature...................

\section{Introduction and background}

One of the most critical decisions in the design of a solar thermal power unit is the choice of the radiation concentration geometry [1] [2], which is in turn connected with the thermal flux needed in the receiver for fulfilling the objectives of the plant. In this context, technical coherence is a major word. In particular, if a high value is chosen for the concentrated radiation thermal flux, the global heat transfer coefficient of the transfer from the receiver surface to the heat carrier fluid must have a similarly high value [1] [2]. Otherwise, the temperature difference between the receiver and the fluid would be very large, which would enhance thermal losses from the receiver. Besides that, large temperatures differences between different parts of the receiver will convey important differential dilation effects, which can be a major cause of concern in the durability of a solar power collector.

There are two main approaches for concentrating solar radiation [1] [2]:

- Parabolic troughs [3] and linear Fresnel concentrators [4], with one axis tracking.

- Revolution parabolic disks and central tower receivers [1], with a two axes tracking system. 
The latter is out of the scope of this article, aimed at analysing the thermal features of linear technologies. The receivers involve a long pipe (or a set of parallel pipes, in a general approach) with a selective coating [5] covering its absorbing surface, i.e., the surface where the concentrated solar radiation impinge. This coating is chosen for having a high absorptivity to solar photons and a low emissivity for photons of the planckian spectrum at similar temperatures to the working ones in the receiver.

Inside the tube, the heat carrier fluid circulates from one end to the other. In most of the cases, collectors are placed in a parallel lay-out, with a high pressure header for distributing the fluid from the central block of the plant (the BOP) and a low pressure header that collects the streams of all the parallel solar receivers and sends the total hot stream into the BOP.

A third line of solar power plant configuration can be cited, the Solar Boiler [6], which is based on a central tower, but without a single central receiver in the top of the tower. The receiver is a bundle of tubes going up along the wall of the tower. This set of tubes can be considered as a linear receiver, although radiation would be concentrated by reflection from a field of heliostats very similar to the central receiver case.

The selective coating is the radiation absorbing element, and it is the component reaching the highest temperature. The useful heat is carried by the fluid and its actual exergy value will depend on the temperature achieved when it leaves from the collector. In some applications, boiling inside the receiver tube is considered, and the steam title will also be a r elevant parameter. In general, the increase in specific enthalpy and the mass flow are the variables characterizing the heating of the fluid. In this context, two energy balances must be taken into account: the overall heating of the fluid, which is equal to the total energy transferred from the absorbing surface to the heat carrier fluid, and the detailed heat transfer balance, which depends on the temperature map attained in the collector due to the transmission processes among the components of the collector, with two main results:

- Heat transfer to the carrier fluid, which is the basis of the overall energy balance

- Losses to the environment, mainly through convection to air and radiation to the surroundings

Heat transfer to the carrier fluid depends on its velocity. The convection coefficient between the tube and the fluid can be increased by increasing its speed, but this fact has other effects, as an increase in pressure drop and pumping power [7] [8]. For liquids, this effect can be of second order as compared to the thermal problem. For gases, the mechanical problem can be as important as the thermal one, and the pumping power needed to keep the gas flow at the level required by the thermal requirement, can be as large as the power collected from the sun, what makes the plant useless.

Another important point in the design of the system is the actual limitations in the temperature of the different components. This is the case of the selective coating, which sets up a ceiling to the maximum temperature allowed in the collector. Coatings resisting $500{ }^{\circ} \mathrm{C}$ without degradation are commercially available, but this is not usually the binding limitation, because in current trough-collector power plants, the most popular heat carrier fluid is synthetic oil, such as Therminol VP1 and the maximum working temperature is below $400{ }^{\circ} \mathrm{C}$.

Those limitations in temperature convey similar limitations in the maximum thermal flux impinging on the receiver, and therefore in the concentration factor. Nevertheless, those 
limitations in the temperature of some materials have to be assessed in the full analysis of the system. If it is effective in transferring the heat to the carrier fluid, and the flow is large enough for guaranteeing a controlled heating, all temperatures will be within the corresponding limits. However, this guarantee relies on the proper circulation of fluid, and it embodies the afore-mentioned problem of the required pumping power.

This coupling of thermal and hydraulics constraints can be characterized in the case of linear collectors, to identify optimum solutions in some design windows, particularly aimed at reducing the cost of solar power plants. This optimization process will embody an exergy analysis of a system featured by the working temperature reached by the heat carrier fluid.

\section{Methodology: A linear collector model}

There are detailed physical and numerical models to calculate trough collectors and reflection Fresnel devices [6] [9] [10]. They are particularly useful to feature a power plant in nominal conditions, which include the set of variables to define the calculation of the collector for the reference conditions chosen as nominal ones, and they can also be used for integrating the performance along the year.

The model used for this study works in similar form to that used in Muñoz et al [6], but does not take into account neither the way used for concentrating the solar radiation, nor the natural aperture of the sun beams, which is an important factor when the concentration geometry involves large distances.

This model can be described as a lumped-parameters representation of the collector. It includes an expression for featuring the useful heat, which is associated with a temperature in the carrier fluid at the collector outlet. The model can be used for investigating the relative importance of some parameters, or equations coefficients, and for identifying relevant trends in the evolution of the system when the boundary conditions change, including the change in the concentration of solar radiation.

The simplicity of the model does not allow using it for designing purposes, or for optimizing a system, but the model is easily followed, unlike many computational codes, which are managed as black boxes. The model is based on the equations listed below, and it can be used to feature general trends in the behaviour of the linear receivers. It is obvious that some hypotheses can not be kept in detailed computations, which are needed for calculating a given design in specified conditions.

The model is an integrated energy balance where the impinging radiation on the receiver goes to useful heat or to thermal losses (convection through the air and radiation to the background). Both thermal phenomena involve several heat transfer mechanisms, but they can be lumped into a single step, expressed in terms of the difference between relevant temperatures, and they include a coefficient, to which an effective value must be assigned. Those values can be estimated from previous detailed calculations of similar systems or from the bibliography. Moreover, sensitivity calculations can easily be carried out by varying those values and other parameters characterizing the boundary conditions.

Four temperatures are sufficient to define the model:

The temperature $T_{c}$ of the absorbing surface (selective coating) where the concentrated radiation impinges. It represents an average value of the coating temperature 
- $\quad$ The temperature of the heat carrier fluid as an average value as it is heated along the receiver, $\mathrm{T}_{\mathrm{f}}$

- $\quad$ The bulk temperature of the air, $\mathrm{T}_{\mathrm{a}}$

- The temperature of the background radiation or environment, $\mathrm{T}_{\text {env. }}$. It can represent either the temperature of the earth's surface, $\mathrm{T}_{\text {grnd }}$, or the temperature of the high atmosphere [1][11], $\mathrm{T}_{\text {sky }}$, each one being affected by their corresponding view factor from the receiver (detailed analysis of this matter demonstrates that for the considered values this dependence has no $r$ elevant effect on the results)

Additional parameters of the model are the following ones. (Values assumed for the first set of calculations are given within brackets.):

- $\quad$ DNI $=$ direct normal irradiation $\left(1 \mathrm{~kW} / \mathrm{m}^{2}\right)$

- $\quad \mathrm{C}_{\mathrm{f}}=$ concentration factor (variable)

- $\quad \varepsilon=$ selective coating emissivity $(0.1)$

- $\quad \alpha=$ selective coating absorptivity (0.9)

- $\quad \mathrm{U}=$ global coefficient for convection losses $\left(8 \mathrm{~W} / \mathrm{m}^{2} \cdot \mathrm{K}\right)$

- $\quad \mathrm{h}=\mathrm{g}$ lobal coefficient for heat transfer from the absorbing surface to the heat carrier fluid $\left(2 \mathrm{~kW} / \mathrm{m}^{2} \cdot \mathrm{K}\right)$

In general, for all calculations the boundary conditions temperatures are:

- $\quad \mathrm{T}_{\text {air }}=25^{\circ} \mathrm{C}(=298 \mathrm{~K})$

- $\quad \mathrm{T}_{\text {grnd }}=25^{\circ} \mathrm{C}(=298 \mathrm{~K})$

- $\quad \mathrm{T}_{\text {sky }}=0.0552 \cdot \mathrm{T}_{\text {air }} 1.5[\mathrm{~K}][11]$

The model is defined by the following equations:

$Q_{\text {sun }}=Q_{\text {conv }}+Q_{\text {rad }}+Q_{\text {use }}$

$Q_{\text {sun }}=C_{f} \cdot \alpha \cdot D N I$

$Q_{\text {conv }}=U \cdot\left(T_{c}-T_{\text {air }}\right)$

$Q_{\text {rad }}=\varepsilon \cdot \sigma \cdot\left(T_{c}^{4}-T_{e n v}^{4}\right)$

$Q_{u s e}=h \cdot\left(T_{c}-T_{\text {use }}\right)$

The absorbed energy and losses that are produced by convection and radiation, adding the cooling effect of the heat transfer fluid (thermal oil) determines the temperature level and the energy efficiency in the receiver itself that can be given, in function of the enthalpy increase in the heat transfer fluid $\left(\mathrm{Q}_{\text {use }}\right)$ by:

$\eta_{\text {th }}=\frac{Q_{\text {use }}}{Q_{\text {sun }}}$

Thermal efficiency is not sufficient to characterize a s ystem designed to yield useful work against the environment: we also use the exergetic efficiency $\left(\eta_{\mathrm{ex}}\right)$ that take into account the 
required blower power $\left(\mathrm{W}_{\mathrm{b}}\right)$ with $\eta_{\mathrm{th} \rightarrow \mathrm{e}}$ is the conversion efficiency from thermal to electrical energy, evaluated as $33 \%$ [6]:

$\eta_{e x}=\frac{Q_{u s e}-W_{b} / \eta_{t h \rightarrow e}}{Q_{\text {sun }}} \cdot\left(1-\frac{T_{a}}{T_{u s e}}\right)$

\section{Results}

This physical model has been applied to some simplified cases with the coefficients already given (first series of calculations) and the results are depicted in Fig. 1 and Fig. 2. It is worth commenting on the shape of the efficiency curve (thermal and exergetic), with two branches that are associated to two thermal regimes: a first branch with rapid increase in efficiency, followed by an almost horizontal second branch, where the heat transfer process from the collector's absorbing surface to the heat carrier fluid is saturated, and the efficiency value reaches a maximum value. A threshold can be marked as the beginning of the second regime. This threshold can be featured by the value of the slope of the curve, or by the relative value to the asymptotic level of the efficiency. In the case $400^{\circ} \mathrm{C}$ of fluid temperature the threshold of the second regime can be marked around a concentration factor of 20. If the fluid temperature is $300{ }^{\circ} \mathrm{C}$, the threshold in the concentration factor is about 15 . W ith a fluid temperature of $1000^{\circ} \mathrm{C}$ the threshold is at 70 .

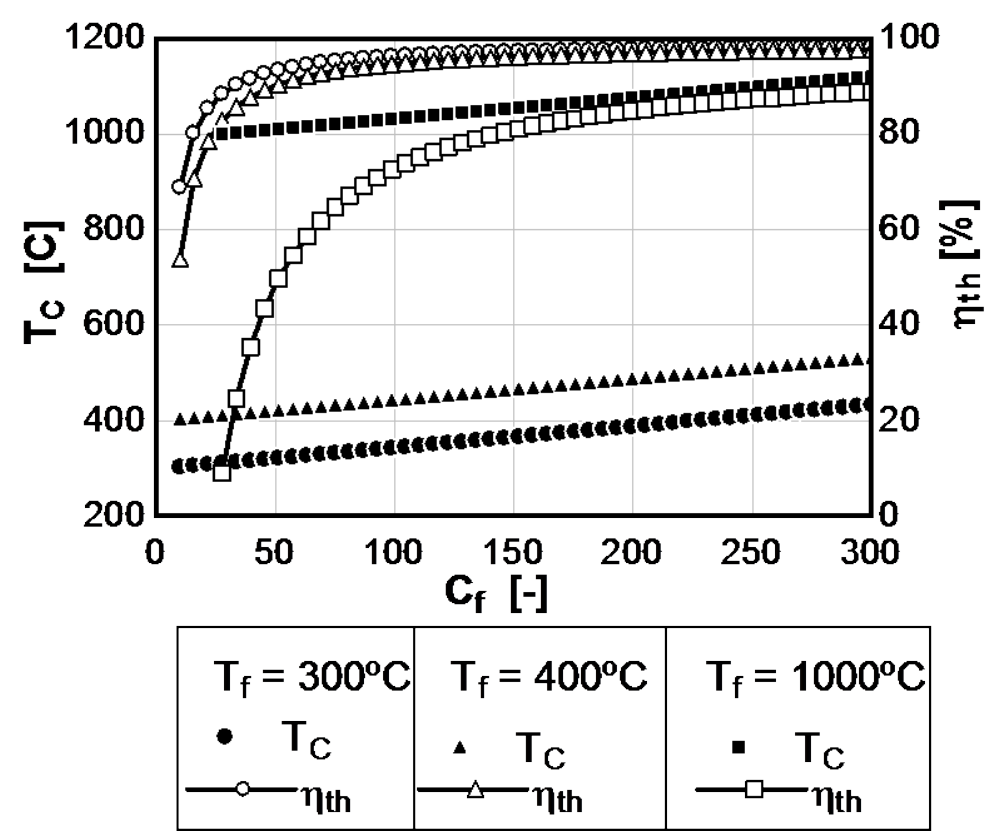

Fig. 1. Collector thermal efficiency and absorbing surface temperature Vs. concentration factor, for three values of $T_{f}$ : $300{ }^{\circ} \mathrm{C}, 400^{\circ} \mathrm{C}$ and $1000^{\circ} \mathrm{C}$. The global coefficient for heat transfer from the absorbing surface to the fluid, " $h$ ", has a value of $400 \mathrm{~W} / \mathrm{m}^{2} \cdot K$

It should be noted as well that the collector's absorbing surface temperature increases linearly with the concentration factor. This effect is influenced by some obvious limitations, because all materials, from the selective coating to the heat carrier fluid, have maximum operational temperatures (which can also depend on the mechanical stresses, in the case of structural materials).

As an initial advice from this analysis, it can be stated that linear collectors should be designed to operate in the saturated regime, limiting the concentration factor in order to limit 
the temperatures. Moreover, the design point can be placed slightly above the threshold for that regime, if this option conveys a significant cost reduction because a lower concentration factor can be achieved with simpler and more robust collectors. Of course, this advice must be properly checked in a co st/benefit analysis, after reviewing in detail the thermo-physical features of collecting solar radiation in linear receivers.

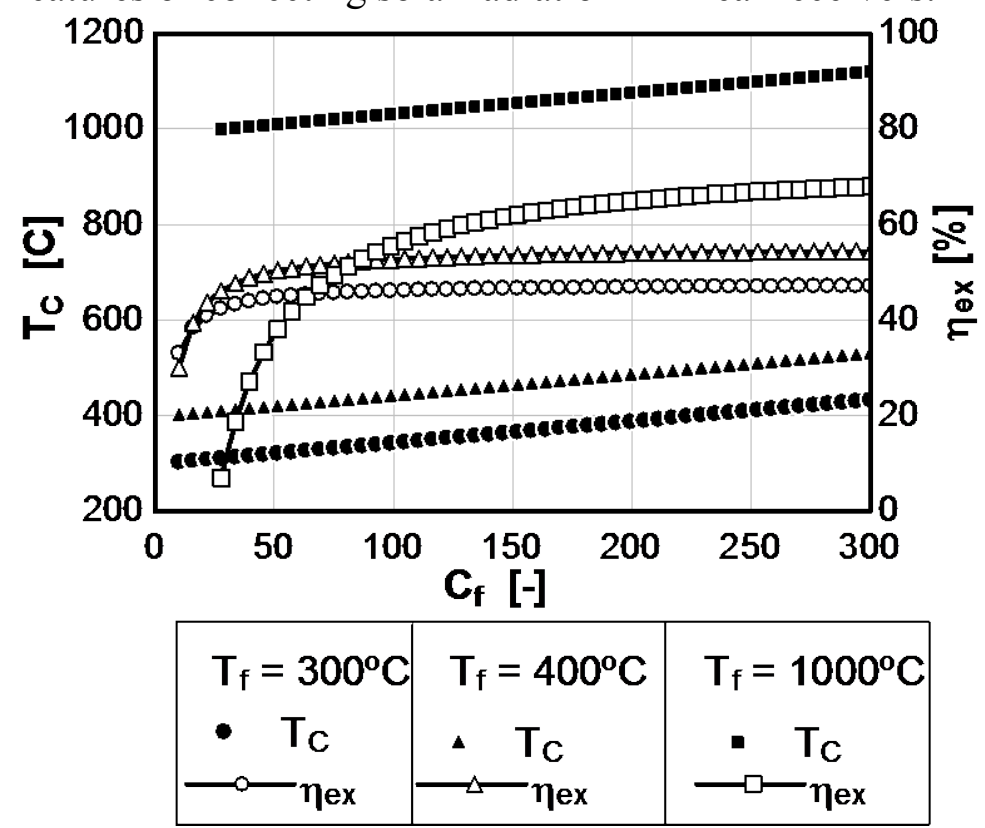

Fig. 2. Collector thermal efficiency and absorbing surface temperature Vs. concentration factor, for three values of $T_{f}: 300{ }^{\circ} \mathrm{C}, 400{ }^{\circ} \mathrm{C}$ and $1000^{\circ} \mathrm{C}$. The global coefficient for heat transfer from the absorbing surface to the fluid, " $h$ ", has a value of $400 \mathrm{~W} / \mathrm{m}^{2} \cdot K$

This finding opens a new way for Concentrated Solar Power, because a Fresnel of large size can be used for heating synthetic oil up to $390^{\circ} \mathrm{C}$, for activating a Rankine cycle similar to those of current power plants with parabolic trough collectors as Fig. 3 shows, with five Fresnel systems, each one of one total length, and all with the same impinging power: $2.5 \mathrm{MW}_{\text {th }}$. The intensity goes from 5 to $25 \mathrm{~kW} / \mathrm{m}^{2}$, corresponding to lengths varying from 500 $\mathrm{m}$ from the former to $100 \mathrm{~m}$ for the latter.

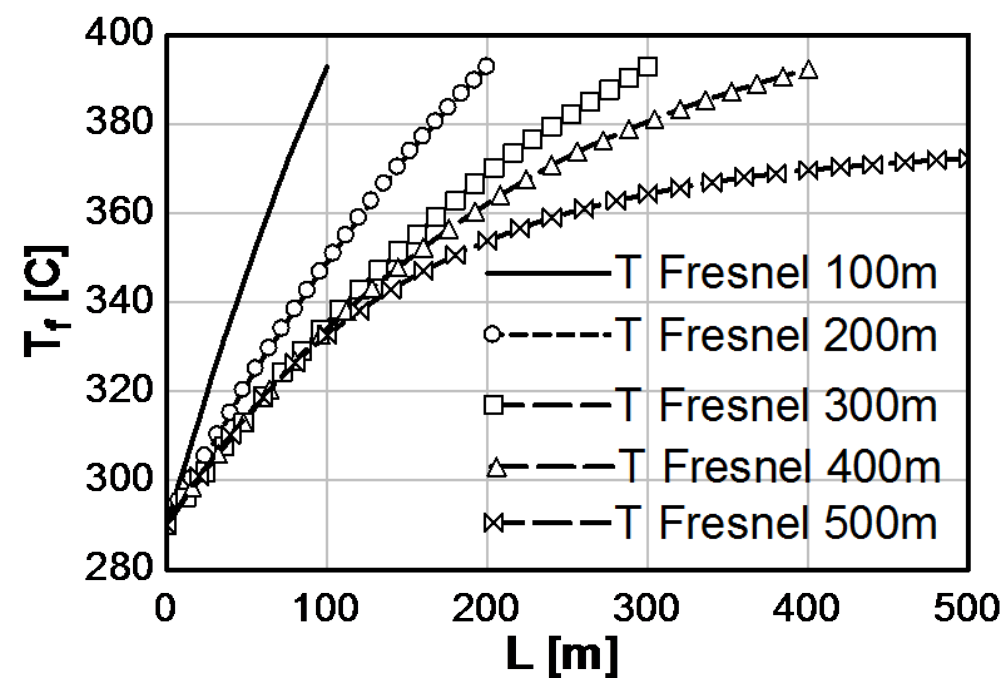

Fig. 3. Evolution of the fluid temperature along the collector length for a set of linear collectors receiving the same total power, with different radiation intensities and lengths. 
It is seen that the case of $5 \mathrm{~kW} / \mathrm{m}^{2}$ does not reach the objective $\mathrm{T}$ ( $392{ }^{\circ} \mathrm{C}$, corresponding to Therminol VP1 as heat transfer fluid), which implies that this case is below the threshold. Energy and exergy efficiencies are shown in Fig. 4, and it is seen how fast they decrease as the radiation intensity goes down. It must be said that this effect is produced, to a large extent, by the objective of achieving a final $\mathrm{T}$ of $392^{\circ} \mathrm{C}$.

It is obvious that requiring higher temperatures in the fluid makes the receiver less efficient, because the temperature gap from the irradiated surface to the bulk of the fluid is shorter than the gap of less demanding collectors, and thermal losses to the surrounding materials increase, as it remains the same. So, if the fluid temperature required for a particular plant is much higher than $400^{\circ} \mathrm{C}$, a Fresnel is not likely to be suitable, because its efficiency will be modest.

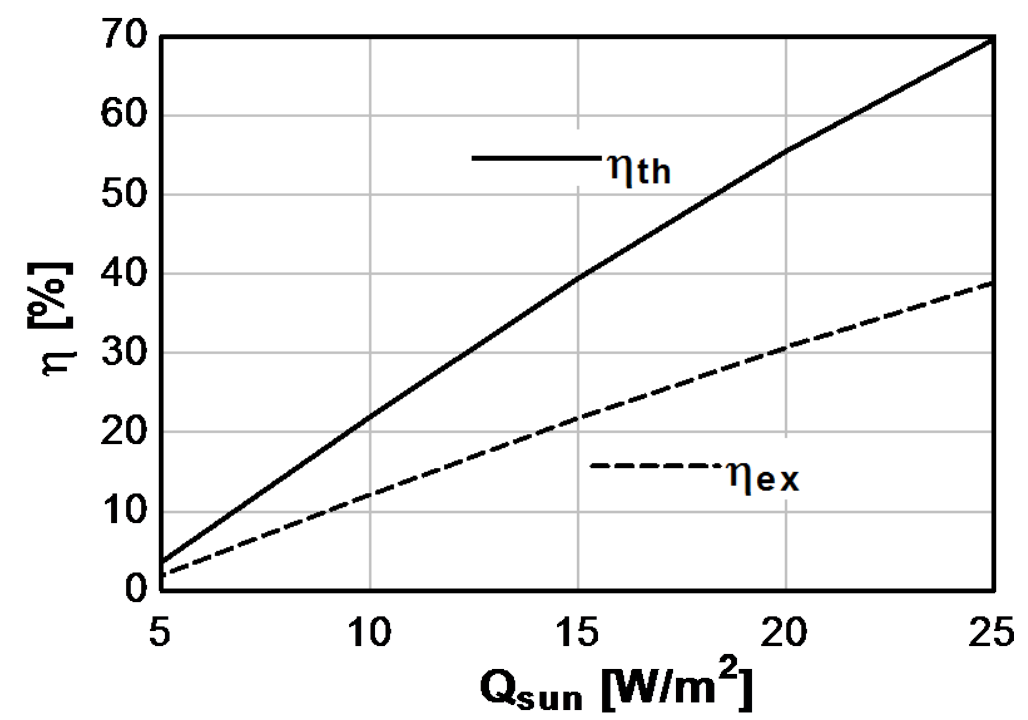

Fig. 4. Energy and exergy efficiencies of the collectors of the set introduced in the previous figure.

\section{Conclusions}

The analysis of thermal performance of linear collectors in the field of concentrated solar power shows the existence of two regimes: a lower regime, corresponding to small values of intensity impinging on the receiver and an upper regime, where the relevant thermal variable (notably, the efficiency) is almost saturated, opposite to the lower regime, where it increases very rapidly as radiation intensity does. The change from the lower to the upper regime is usually very well identified by a value, or a s hort range of values. It exists therefore a threshold for reaching the saturated regime. All these features depend on the temperature required in the heat-carrier fluid.

In this performance analysis it has been found that moderate concentration factors can be close to optimal values, due to temperature constraints. Thermal and exergetic efficiency curves show that beyond these values, i.e. increasing the concentration factor, the threshold is exceeded, and therefore efficiency increases slowly.

In the specific case of $400^{\circ} \mathrm{C}$ as objective temperature - usual in current solar thermal plants with through collectors that uses thermal oil - the concentration factor takes the value 20 approximately.

This observation must be properly exploited by identifying the simplest and most robust concentration scheme able to attain the upper regime of a given application, for feeding the 
boiler of a Rankine cycle with given working temperatures. This is the case of linear Fresnel reflecting collectors, which avoid mobile parts such as ball-joints and flexible joints that could be important leak-points, as well as metal-glass welds, which are another cause of failure in trough collectors

\section{References}

[1] J.A. Duffie \& W.A. Beckman, Solar Engineering of Thermal Processes, John Wiley and Sons, New york, 2nd ed., 1991, pp. 330-378

[2] D. Mills, Advances in solar thermal electricity technology, Solar Energy, vol. 76, no. 1-3, 2004, pp. 19-31

[3] A. Fernández-García, E. Zarza, L. Valenzuela \& M. Pérez, Parabolic-trough solar collectors and their applications, Renewable and Sustainable Energy Reviews, vol. 14, no. 7, 2010, pp. 1695-1721

[4] D.R. Mills \& G.L. Morrison, Compact Linear Fresnel Reflector solar thermal powerplants, Solar Energy, vol. 68, no. 3, 2000, pp. 263-283

[5] M. Farooq \& I.A. Raja, Optimisation of metal sputtered and electroplated substrates for solar selective coatings, Renewable Energy, vol. 33, no. 6, 2008, pp. 1275-1285

[6] J. Muñoz, A. Abánades \& J.M. Martínez-Val, A conceptual design of solar boiler, Solar Energy, vol. 83, no. 9, 2009, pp. 1713-1722

[7] F.P. Incropera, Fundamentals of heat and mass transfer, John Wiley \& Sons, Danvers, USA, 6th ed, 2007, pp 485-517

[8] R.L. Mott, Applied fluid mechanics, 6th ed., Pearson Prentice Hall, Upper Saddle River, 2006, pp 226-245

[9] R. Forristal, Heat Transfer Analysis and Modelling of a Parabolic Trough Solar Receiver Implemented in Engineering Equation Solver, NREL Technical Report, Golden, Colorado, USA, 2003

[10]M.J. Montes, A. Abánades \& J.M. Martínez-Val, Performance of a direct steam generation solar thermal power plant for electricity production as a function of the solar multiple, Solar Energy, vol. 83, no. 5, 2009, pp. 679-689

[11]W.C. Swinbank, Long-wave radiation from clear skies, Quarterly Journal of the Royal Meteorological Society, vol. 89, no. 381, 1963, pp. 339-348

[12] J.M. Martínez-Val, Thermal solar energy collector, Es 2231 576, P CT/ES2009/000557, WO2010/076350 08 July 2010 (08.07.2010) 